\title{
An imaging system with calibrated color image acquisition for use in dermatology
}

\author{
Yves Vander Haeghen, J.M. Naeyaert, I. Lemahieu (IEEE Member), W. Philips (IEEE Member)
}

\begin{abstract}
We propose a novel imaging system useful in dermatology, more precisely for the follow-up of patients with an increased risk of skin cancer. The system consists of a Pentium PC equipped with a RGB frame grabber, a 3chip CCD camera controlled by the serial port and equipped with a zoom lens and a halogen annular light source.

Calibration of the imaging system provides a way to transform the acquired images, which are defined in an unknown color space, to a standard well-defined color space called sRGB. sRGB has a known relation to the $\mathrm{CIE}^{1} \mathrm{XYZ}$ and CIE $L^{*} a^{*} b^{*}$ colorimetric spaces. These CIE color spaces are based on the human vision, and allow the computation of a color difference metric called CIE $\Delta \mathrm{E}_{\mathrm{ab}}^{*}$, which is proportional to the color difference as seen by a human observer. Several types of polynomial RGB to sRGB transforms will be tried, including some optimized in perceptually uniform color spaces. The use of a standard and well-defined color space also allows meaningful exchange of images, e.g. in teledermatology.

The calibration procedure is based on 24 patches with known color properties, and takes about 5 minutes to perform. It results in a number of settings called a profile which remains valid for tens of hours of operation. Such a profile is checked prior to acquiring images using just one color patch, and is adjusted on the fly to compensate for short-term drift in the response of the imaging system. Precision or reproducibility of subsequent color measurements is very good with $\left\langle\Delta \mathrm{E}_{\mathrm{ab}}^{*}\right\rangle=0.3$ and $\Delta \mathrm{E}_{\mathrm{ab}}^{*}<1.2$. Accuracy compared to spectrophotometric measurements is fair with $\left\langle\Delta \mathrm{E}_{\mathrm{ab}}^{*}\right\rangle=6.2$ and $\Delta \mathrm{E}_{\mathrm{ab}}^{*}<\mathbf{1 3 . 3}$.
\end{abstract}

Index-terms - Dermatology, Color imaging, Imaging system calibration, Colorimetry, CIE

\section{INTRODUCTION}

$\mathrm{I}^{\mathrm{N}}$ $\mathrm{N}$ dermatology, color and color difference as well as shape often convey important diagnostic information. Although visual inspection by the dermatologist goes a long way, quantitative color and shape measurements might be helpful when investigating pigmented lesions and especially skin cancer where early diagnosis is crucial but quite difficult. In the follow-up of patients with an increased risk of developing skin cancer, the evolution of a suspect skin lesion also plays an important role, so a visual record is primordial.

Such a visual record is generally obtained using traditional photography. Unfortunately, it is unsuited for quan-

Y. Vander Haeghen is with the Dept. Engineering, ELIS-MEDISIP, University Gent, St.-Pietersnieuwstraat 41, 9000 Gent, Belgium, and the Dept. Dermatology, University Hospital, De Pintelaan 185, 9000 Gent, Belgium. Email: Yves.VanderHaeghen@rug.ac.be

J.M. Naeyaert is with the Dept. Dermatology, University Hospital, De Pintelaan 185, 9000 Gent, Belgium

I. Lemahieu is with the Dept. Engineering, ELIS-MEDISIP, University Gent, St.-Pietersnieuwstraat 41, 9000 Gent, Belgium

W. Philips is with the Dept. of Telecommunications and Information Processing, University Gent, St. -Pietersnieuwstraat 41, 9000 Gent, Belgium

1'Commission Internationale de l'Eclairage' a standardizing body in the field of color science titative color measurements due to variations in, e.g., film and development. On the other hand, chroma- and spectrophotometers allow accurate color measurements. The drawback with these instruments is that they take average measurements over a fixed area and are unable to measure the irregular shapes often found in skin lesions. They also do not provide a visual record of the lesion.

The use of digital imaging in dermatology has already been investigated several times, mostly using color spaces like $R G B$ and related spaces like $H S V$ [1], [2], [3], [4]. The problem with this approach is that there are literally thousands of different $R G B$ spaces because $R G B$ is dependent on the imaging system, i.e. it is a device-dependent color space. Consequently, developed methods and obtained results may suffer from a limited applicability. It also means cross-validation of methods and meaningful exchange of acquired images are very difficult. Another problem with this approach is that it is very hard to relate differences between colors in these device-dependent color spaces with the perception of such color differences by a human. Such perceptual color differences can be very useful in e.g. segmentation, and are generally computed with the aid of a socalled 'perceptually uniform color space', e.g. CIE $L^{*} a^{*} b^{*}$. In [5], [6] these perceptual color differences were used, but as far as can be made out the imaging system $R G B$ values were transformed using a standard CIE transform, which is only valid for a well-defined $R G B$ space and generally not applicable.

As hinted on in the previous paragraph, digital imaging suffers some of the same drawbacks as traditional photography [7], [8]: a non-reproducible device-dependent color representation, usually $R G B$. Basically, each (digital) imaging system has its own time-varying $R G B$ color space, depending, amongst others, on the spectral sensitivities of its color sensors, its settings, the temperature, etc. It is possible to calibrate such an imaging system by controlling its settings and determining the relationship between its unknown device-dependent color space, and some deviceindependent color space. The problem of finding this transform has already been covered extensively in the literature [9], [10], [8], [11], [12], [13], [14], [15].

In this paper, however, the complete calibration and acquisition procedure of a specific imaging system build for a specific task will be detailed. The choice of the central device-independent color space of the imaging system and its relation to other color spaces, as well as its advantages and disadvantages are discussed in section II. Next, the model of the imaging system on which the calibration procedure is based is introduced in IV-A. This is followed by a more detailed overview in IV-B of the polynomial trans- 
forms converting device-dependent to device-independent color values which were tried and evaluated. Some of these transforms were optimized iteratively in a perceptually uniform color space as opposed to the more usual transform being a least-squares solution of an overdetermined set of linear equations in the device-independent color space. Section $\mathrm{V}$ is devoted to the different steps in the calibration and the acquisition procedure, which must be practical and easy to perform. The calibration procedure only has to be performed after a few tens of hours of normal operation, and requires minimal user interaction. During the acquisition procedure the calibration settings are checked and adjusted on the fly using just one color patch, and subsequently calibrated images may be acquired for about 10-15 minutes before a new check and adjustment is necessary. Thereafter, the precision and accuracy are discussed in section VI, and some conclusions are drawn.

\section{IMAGING SYSTEM COLOR SPACES}

Color spaces play a central role in the development of the imaging system, so we will elaborate on this matter briefly before moving on. For more background information we refer to [16] and [17].

As mentioned in the previous section, calibration mainly consists of controlling the settings of the imaging system and determining the relationship between its unknown, usually $R G B$ like, device-dependent color space to some device-independent color space. We chose the standard $s R G B$ color space as this device-independent color space and will now discuss some of the repercussions of this decision (see fig. 1).

The $s R G B$ color space is based on the phosphors used in many modern CRT-based display devices, including computer monitors. This means that an image stored in $s R G B$ doesn't have to be converted before display, and should look fairly realistic on a computer monitor. $s R G B$ has a white point of $6500 \mathrm{~K}$ color temperature or D65, which means that the color produced by combining the full output of each color channel on an $s R G B$ output device is the same as that of a black body at $6500 \mathrm{~K}$. It is the subject of standardization, so meaningful exchange of images is also possible. $s R G B$ tristimulus values $(\mathcal{R}, \mathcal{G}, \mathcal{B})$ have a known relationship to CIE $X Y Z$ tristimulus values $(X, Y, Z)$, a human vision based color space:

$$
\begin{aligned}
\left(\begin{array}{l}
X \\
Y \\
Z
\end{array}\right) & =\Phi_{s R G B \rightarrow X Y Z}\left(\begin{array}{l}
\mathcal{R} \\
\mathcal{G} \\
\mathcal{B}
\end{array}\right) \\
& =100\left(\begin{array}{lll}
0.412453 & 0.357580 & 0.180432 \\
0.212671 & 0.715160 & 0.072169 \\
0.019334 & 0.119193 & 0.950227
\end{array}\right)\left(\begin{array}{l}
\mathcal{R} \\
\mathcal{G} \\
\mathcal{B}
\end{array}\right)
\end{aligned}
$$

The $s R G B$ component values are comprised between 0 and 1, while for the $X Y Z$ values a luminance of 100 , i.e. $Y=100$, represents a perfect white diffuser. The inverse transform $\Phi_{X Y Z \rightarrow s R G B}=\Phi_{s R G B \rightarrow X Y Z}^{-1}$ can easily be determined using matrix inversion.

CIE $L^{*} a^{*} b^{*}$ color triplets $\left(L^{*}, a^{*}, b^{*}\right)$ are a non-linear transformation of CIE $X Y Z$ tristimulus values $(X, Y, Z)$ and are an attempt at a perceptually uniform color space:

$$
\begin{aligned}
L^{*} & =116 f\left(Y / Y_{w}\right)-16 \\
a^{*} & =500\left(f\left(X / X_{w}\right)-f\left(Y / Y_{w}\right)\right) \\
b^{*} & =200\left(f\left(Y / Y_{w}\right)-f\left(Z / Z_{w}\right)\right) \\
f(t) & = \begin{cases}7.787 t+\frac{16}{116} & \text { if } t<0.008856 \\
t^{1 / 3} & \text { if } 0.008856 \leq t \leq 1 .\end{cases}
\end{aligned}
$$

Typical ranges for CIE $L^{*} a^{*} b^{*}$ color triplets is $L^{*} \in$ $[0,100], a^{*} \in[-100,100]$ and $b^{*} \in[-100,100]$. $\left(X_{w}, Y_{w}, Z_{w}\right)$ is the tristimulus of the white point under which the colors are observed. Here this is the same as the D65 white point of the $s R G B$ space, given by $(95.017,100.0,108.813)$. The division of the tristimulus $(X, Y, Z)$ by the tristimulus of the white point models a physiological phenomenon of the HVS which is called chromatic adaptation. This adaptation of the visual pathways ensures a light source appears more or less 'white' after a little while, whether it is more yellowish like tungsten lighting, or more bluish like sunlight from a slightly overcast sky. By construction the Euclidean distance between two colors $\left(L_{1}^{*}, a_{1}^{*}, b_{1}^{*}\right)$ and $\left(L_{2}^{*}, a_{2}^{*}, b_{2}^{*}\right)$, notated as $\Delta E_{a b}^{*}$, is more or less proportional to their color difference as perceived by a human observer:

$$
\Delta E_{a b}^{*}=\sqrt{\left(L_{1}^{*}-L_{2}^{*}\right)^{2}+\left(a_{1}^{*}-a_{2}^{*}\right)^{2}+\left(b_{1}^{*}-b_{2}^{*}\right)^{2}}
$$

To put this color difference metric in perspective we may say that the theoretical just noticeable difference is about $1 \Delta E_{a b}^{*}$ unit, but usually anything below $3 \Delta E_{a b}^{*}$ units goes unnoticed.

For convenience we also introduce the non-linear operator $\Phi_{s R G B \rightarrow L^{*} a^{*} b^{*}}$ which represents the transform from $s R G B$ tristimulus values to $\operatorname{CIE} L^{*} a^{*} b^{*}$ via the CIE $X Y Z$ color space:

$$
\begin{aligned}
\left(\begin{array}{l}
L^{*} \\
a^{*} \\
b^{*}
\end{array}\right) & =\Phi_{s R G B \rightarrow L^{*} a^{*} b^{*}}\left(\begin{array}{l}
\mathcal{R} \\
\mathcal{G} \\
\mathcal{B}
\end{array}\right) \\
& =\Phi_{X Z Y \rightarrow L^{*} a^{*} b^{*}} \Phi_{s R G B \rightarrow X Y Z}\left(\begin{array}{l}
\mathcal{R} \\
\mathcal{G} \\
\mathcal{B}
\end{array}\right) .
\end{aligned}
$$

$\Phi_{X Z Y \rightarrow L^{*} a^{*} b^{*}}$ represents a non-linear operator implementation of the equations in (2), the precise form of which is not important here. Note that again this operator is perfectly invertible, leading to the CIE $L^{*} a^{*} b^{*}$ to $s R G B$ transform $\Phi_{L^{*} a^{*} b^{*} \rightarrow s R G B}=\Phi_{s R G B \rightarrow L^{*} a^{*} b^{*}}^{-1}$ :

$$
\left(\begin{array}{l}
\mathcal{R} \\
\mathcal{G} \\
\mathcal{B}
\end{array}\right)=\Phi_{L^{*} a^{*} b^{*} \rightarrow s R G B}\left(\begin{array}{l}
L^{*} \\
a^{*} \\
b^{*}
\end{array}\right) .
$$

The $s R G B$ and its related CIE color spaces provide a proper framework for subsequent development of segmentation, measuring and classification methods (see again fig. 1). Especially segmentation should benefit from the availability of a perceptual color difference and produce results which are more in agreement with a human observer. Moreover, any such segmentation, measurement or classification method should have a wider applicability due to its independence from an imaging system. It will also allow independent testing of these methods and opens up new possibilities in teledermatology. 


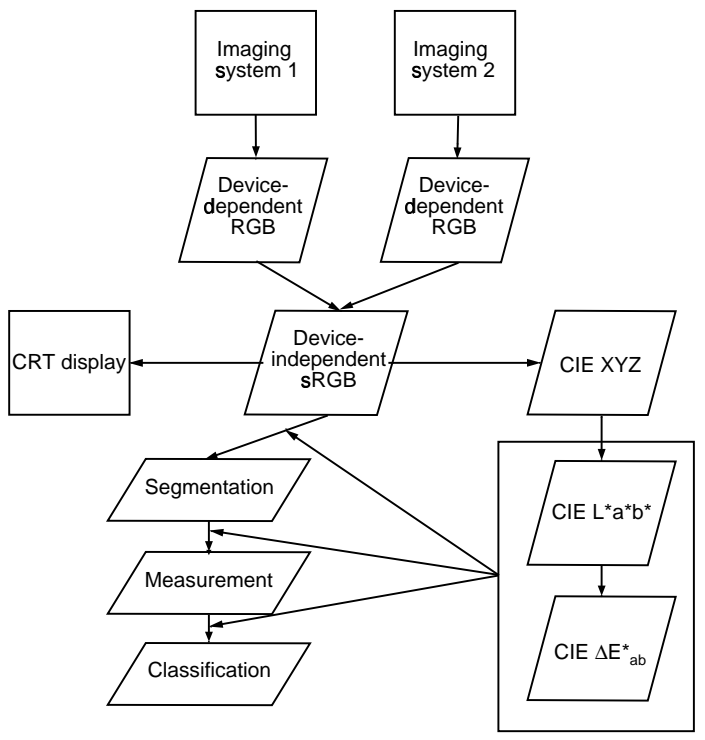

Fig. 1. Schematic view of the role of the $s R G B$ color space and its relationship with other color spaces.

One disadvantage of $s R G B$ is its rather limited gamut compared to that of the human visual system (HVS), which is why CIE $X Y Z$ is generally chosen as the deviceindependent space when calibrating an imaging device. Luckily, this fact impairs mainly measurement and reproduction of the very saturated, vivid colors, and these are not very abundant in skin imaging.

\section{MATERIALS}

The imaging system consists of a JVC KY-55B 3-chip CCD camera with a Pentax manual zoom lens, a Schott ${ }^{2}$ KL1500 150 Watt halogen light source and an Integral Technologies $^{3}$, FlashPoint 128 frame grabber.

The core of the imaging system is a $\mathrm{JVC}^{4} \mathrm{KY}-55 \mathrm{~B}$ 3-chip CCD camera, which can be remote controlled using the serial port of a PC. It is equipped with a Pentax manual zoom lens and its field of view is set to $1.6 \mathrm{~cm}$ by $1.2 \mathrm{~cm}$. With an image containing 760 by 570 pixels the resolution is thus 47.5 pixels $/ \mathrm{mm}$. Proper lighting is crucial in acquiring high-quality and reproducible images, and in order to obtain a spatially homogeneous light field a Schott continuous annular light diffuser is fitted around the lens. This diffuser also ensures that the angle of the incident light on the focal plane is different from 90 degrees, thereby avoiding or at least diminishing specular, i.e. mirror-type, reflections. The diffuser is connected to a Shott KL1500 150 Watt halogen light source using a $2 \mathrm{~m}$ optical fiber. This source operates continuously (as opposed to a flash type operation) but at high frequency so that no light intensity variations over time are apparent, even at 30 acquisition rates of frames per second. An extra blue filter in the light path then changes the color temperature from $2800 \mathrm{~K}$ to

\footnotetext{
${ }^{2}$ Shott Glaswerke, Hagenauer strasze 38, D-65203 Wiesbaden

${ }^{3}$ Integral Technologies, Inc, 9855 Crosspoint Blvd, Suite 126, Indianapolis, Indiana, 46256 USA

${ }^{4}$ JVC: Victor Company of Japan, limited
}

$6500 \mathrm{~K}$, which makes it a very rough simulation of a CIE D65 illuminant and the white point of the $s R G B$ color space.

The penetration of stray light in the lens must of course be avoided, and so a black cardboard cylinder with a glass frontplate is fixed to the annular diffuser. The glass frontplate makes sure the skin lesion is in the focal plane of the lens, and can be used for dermatoscopy, also called epiluminscence microscopy (ELM), by applying some oil onto it before pressing it down on the skin lesion. This diminishes the refraction index mismatch at the glass-skin interface, and has the effect of making the horny layer of the skin somewhat transparent, thereby exposing some diagnostically relevant deeper structures. Calibration of the imaging system for dermatoscopy would be difficult and time consuming, and so will not be attempted. Rather, it is hoped that if the same oil is used in the same quantities calibration for clinical imaging is sufficient to ensure accurate and reproducible dermatoscopic imaging.

Importantly, the glass frontplate which comes in contact with the patient can also be desinfected. Ideally this plate should be equipped with some kind of pressure measuring device, as it is known that skin color may vary strongly with applied pressure, but this is not the case for the moment being. Finally, to make handling easier the camera is equipped with a pistol grip with a trigger. The trigger is connected to the frame grabber and controls image acquisition. See fig. 2 for a $3 \mathrm{D}$ representation of the camera assembly.

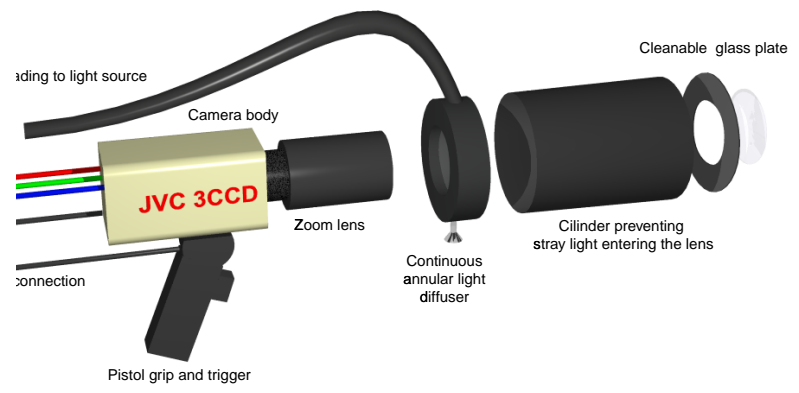

Fig. 2. Schematical drawing of the camera and its accessories. All components are drawn approximately to scale.

The Integral technologies ${ }^{5}$ Flashpoint 128 frame grabber is responsible for digitizing the video signals sent to it by the camera. It is fitted in a standard $150 \mathrm{MHz}$ Pentium PC running Windows NT 4.0, and acquisition is done using the PAL analog RGB format which is digitized with 8-bit precision per color channel. The settings of the frame grabber are controlled using the FlashPoint Software Developer's Toolkit 3.0 from Integral Technologies. The color patches used in the calibration are taken from the MacBeth Color Checker Chart ${ }^{6}$ (MBCCC).

\footnotetext{
${ }^{5}$ Integral Technologies, Inc, 9855 Crosspoint Blvd, Suite 126, Indianapolis, Indiana, 46256 USA

${ }^{6}$ Macbeth, 405 Little Britain rd, New Windsor, NY 12553-6148, USA
} 
They were precisely measured using a Gretag ${ }^{7}$ SPM50 spectrophotometer (SPM), resulting in CIE $L^{*} a^{*} b^{*}$ color triplets $\left(L_{S P M, i}^{*}, a_{S P M, i}^{*}, b_{S P M, i}^{*}\right), i=1, \ldots, 24$.

\section{IMAGING SYSTEM MODELS AND THEORY}

First we discuss the model on which the calibration of the imaging system is based. Thereafter, the $R G B$ to $s R G B$ transform will be discussed. In what follows the symbol $P=R, G, B$ and $\mathcal{P}=\mathcal{R}, \mathcal{G}, \mathcal{B}$ will be used to indicate both a tristimulus value component and a color channel when used as a subscript, respectively in the $R G B$ and the $s R G B$ color space.

\section{A. The imaging system model}

The two main devices in the imaging system are the CCD-camera and the frame grabber. The camera output voltage $V_{P}^{\text {cam }}$ of the camera for a certain pixel and color channel $P=R, G, B$ can be written as:

$$
V_{P}^{c a m}=\Psi_{0}^{V_{\text {max }}^{c a m}}\left(\Gamma\left(V_{\text {offset }}^{\text {cam }}+g_{P}^{c a m}\left(V_{P}^{C C D}+V_{\text {offset }}^{C C D}\right)\right)\right),
$$

with $V_{\text {offset }}^{\text {cam }}$ the settable camera offset voltage, and $V_{P}^{C C D}$ a voltage proportional to the light incident on the element corresponding with the pixel in CCD array of color channel $P . V_{o f f s e t}^{C C D}$ is an unknown offset voltage resulting from, amongst others, the very temperature sensitive CCD dark current. $g_{P}^{c a m}$ is a variable color channel dependent gain. In our case the gain for the green channel is always 1 , which is why the green channel will be used in determining color channel independent parameters. The function $\Psi_{0}^{V_{\max }^{\text {cam }}}$ represents the clipping that occurs when a voltage is out of range:

$$
\Psi_{0}^{V_{\max }}(V)= \begin{cases}0 & \text { if } V<0 \\ V & \text { if } 0 \leq V \leq V_{\max } \\ V_{\max } & \text { if } V>V_{\max } .\end{cases}
$$

The function $\Gamma$ is called the gamma correction, and is a compensation for the transfer function of a CRT relating electron gun input voltage and light intensity. It allows the camera to drive a CRT directly and can generally be turned on or off. Although we have to invert this operation numerically after digitization in the frame grabber, it is much better to digitize gamma-corrected $R^{*} G^{*} B^{*}$ signals than linear-light $R G B$ when the digitization resolution is less that about 12 to 14 bits [18]. For the same reason it is not a good idea to store $R G B$ or $s R G B$ images in memory or on file, as this would waste a lot of space. Rather, it is much better to store $R^{*} G^{*} B^{*}$ and $s R^{*} G^{*} B^{*}$ values for which 8 bits per component are sufficient, and transform them on the fly if necessary. For $P=R, G, B \in[0,1], \Gamma$ is defined as:

$$
P^{*}=\Gamma(P)= \begin{cases}4.5 P & 0 \leq P \leq 0.018 \\ 1.099(P)^{0.45}-0.099 & 0.018<P \leq 1.0\end{cases}
$$

resulting in gamma-corrected values $P^{*}=R^{*}, G^{*}, B^{*} \in$ $[0,1]$ which are then eventually scaled and rounded to

\footnotetext{
${ }^{7}$ GretagMacbeth AG, Althardstrasse 70, CH-8105 Regensdorf Switserland
}

$\{0, \ldots, 255\}$. This function is easily inverted. The output voltage of the frame grabber before the $\mathrm{AD}$-convertor $V_{P}^{f g}$, the 8-bit digitized $\mathrm{R}^{*} \mathrm{G}^{*} \mathrm{~B}^{*}$ value $P^{*}$ and the linear RGB value $P$ may thus be written as:

$$
\begin{aligned}
V_{P}^{f g} & =\Psi_{0}^{V_{\max }^{f g}}\left(V_{o f f s e t}^{f g}+g^{f g} V_{P}^{c a m}\right) \\
P^{*} & =\Re\left(255 \frac{V_{P}^{f g}}{V_{\max }^{f g}}\right), P^{*} \in\{0, \ldots, 255\} . \\
P & =\Gamma^{-1}\left(\frac{P^{*}}{255}\right), P \in[0,1] .
\end{aligned}
$$

with $V_{\text {offset }}^{f g}$ again a settable offset voltage, $g^{f g}$ the variable frame grabber gain and $\Re$ the integer rounding operator.

In reality, equations (6) and (9) depend on more variable parameters, but these are not relevant to the proposed scheme. The camera parameters $V_{\text {offset }}^{\text {cam }}$ and $g_{P}^{\text {cam }}$ can be set using values in the range $[0,255]$, while the frame grabber values for $V_{\text {offset }}^{f g}$ and $g^{f g}$ have to be in the range $[0,63]$. The relationship between these settings and the actual voltages or gains is supposedly linear.

\section{B. The RGB to sRGB transform}

If the camera sensor spectral sensitivities are equal to or a linear combination of the spectral sensitivities of the HVS then the relationship between the $R G B$ (under D65 lighting) and the $s R G B$ color space would simply be linear. This is generally not the case, and leads to a type of metamerism in which two colors which look the same as seen by the camera (same $R G B$ values) may look different as seen by a human observer (different $X Y Z$ values) under the same lighting conditions. It is clear that although we can try to model the non-linear relationship between the $R G B$ and $s R G B$ color space with higher-order polynomial transforms we will not be able to compensate for this type of machine-human metamerism.

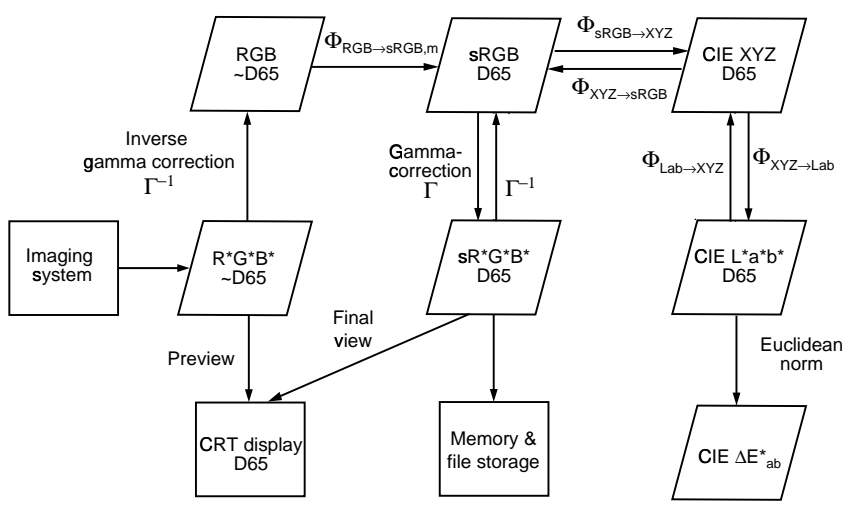

Fig. 3. A more detailed view of the color spaces of the imaging system and their relations between themselves and with imaging system devices.

The $R G B$ to $s R G B$ transform $\Phi$ with $m$ terms can be 
written as (see also fig. 3):

$$
\begin{aligned}
\left(\begin{array}{l}
\mathcal{R} \\
\mathcal{G} \\
\mathcal{B}
\end{array}\right) & =\Phi_{R G B \rightarrow s R G B, m}\left(\begin{array}{l}
R \\
G \\
B
\end{array}\right) \\
& =\left(\begin{array}{lll}
a_{1, \mathcal{R}} & \ldots & a_{m, \mathcal{R}} \\
a_{1, \mathcal{G}} & \ldots & a_{m, \mathcal{G}} \\
a_{1, \mathcal{B}} & \ldots & a_{m, \mathcal{B}}
\end{array}\right)\left[\Theta_{m}\left(\begin{array}{l}
R \\
G \\
B
\end{array}\right)\right]
\end{aligned}
$$

The 'delinearizing' operator $\Theta_{m}$ transforms a 3 element column vector to an $m$ element column vector representing a set of plausible polynomial transforms [15]:

$$
\begin{aligned}
& \Theta_{3}\left(\begin{array}{l}
R \\
G \\
B
\end{array}\right)=\left(\begin{array}{lll}
R & G & B
\end{array}\right)^{t} \\
& \Theta_{6}\left(\begin{array}{l}
R \\
G \\
B
\end{array}\right)=\left(\begin{array}{llllll}
R & G & B & R G & G B & B R
\end{array}\right)^{t} \\
& \Theta_{8}\left(\begin{array}{l}
R \\
G \\
B
\end{array}\right)=\left(\begin{array}{llllllll}
1 & R & G & B & R G & G B & B R & R G B
\end{array}\right)^{t} \\
& \Theta_{9}\left(\begin{array}{l}
R \\
G \\
B
\end{array}\right)=\left(\begin{array}{lllllllll}
R & G & B & R G & G B & B R & R^{2} & G^{2} & B^{2}
\end{array}\right)^{t} \\
& \Theta_{11}\left(\begin{array}{l}
R \\
G \\
B
\end{array}\right)=\left(\begin{array}{llllllllllll}
1 & R & G & B & R G & G B & B R & R^{2} & G^{2} & B^{2} & R G B
\end{array}\right)^{t}
\end{aligned}
$$

The coefficients of the transform $\Phi_{R G B \rightarrow s R G B, m}$ can be determined using the SPM CIE $L^{*} a^{*} b^{*}$ measurements of the 24 MBCCC patches, after transformation to $s R G B$ using eq. (5). The resulting tristimulus values $\left(\mathcal{R}_{S P M, i}, \mathcal{G}_{S P M, i}, \mathcal{B}_{S P M, i}\right), i=1, \ldots, 24$ are then substituted on the left in eq. (10). Next, these patches are measured by the imaging system, and the resulting spatially averaged $R G B$ tristimulus values $\left(R_{i}, G_{i}, B_{i}\right), i=1, \ldots, 24$ are substituted on the right in eq. (10). This results in one set of overdetermined linear equations for each color channel $\mathcal{P}=\mathcal{R}, \mathcal{G}, \mathcal{B}$ of the $s R G B$ color space:

$$
\begin{aligned}
& \left(\begin{array}{lll}
\mathcal{P}_{S P M, 1} & \ldots & \mathcal{P}_{S P M, 24}
\end{array}\right)= \\
& \left(\begin{array}{lll}
a_{1, \mathcal{P}} & \ldots & a_{m, \mathcal{P}}
\end{array}\right)\left(\Theta_{m}\left(\begin{array}{l}
R_{1} \\
G_{1} \\
B_{1}
\end{array}\right) \quad \ldots \quad \Theta_{m}\left(\begin{array}{l}
R_{24} \\
G_{24} \\
B_{24}
\end{array}\right)\right) .
\end{aligned}
$$

These equations can easily be solved in a least-squares sense using e.g. singular-value decomposition (SVD). The resulting transform $\Phi_{R G B \rightarrow s R G B, m}^{L L S Q}$ is called the linear leastsquares (LLSQ) solution. Unfortunately, this solution is only optimal in the mathematical sense, as the sum of squares criterion in the $s R G B$ color space is hardly representative for the human perception of the mapping error associated with the transform $\Phi_{R G B \rightarrow s R G B, m}^{L L S Q}$. Such a 'perceptual' criterion, however, could be based on the color difference metric defined in CIE $L^{*} a^{*} b^{*}$ space, see eq. (3). In order to compute such a criterion we first need to transform the imaging system measurements $\left(R_{i}, G_{i}, B_{i}\right), i=$ $1, \ldots, 24$ of the MBCCC patches to CIE $L^{*} a^{*} b^{*}$ color triplets $\left(L_{i}^{*}, a_{i}^{*}, b_{i}^{*}\right)$ using eqs. (10) and (4):

$$
\left(\begin{array}{c}
L_{i}^{*} \\
a_{i}^{*} \\
b_{i}^{*}
\end{array}\right)=\Phi_{s R G B \rightarrow L^{*} a^{*} b^{*}} \Phi_{R G B \rightarrow s R G B, m}\left(\begin{array}{c}
R_{i} \\
G_{i} \\
B_{i}
\end{array}\right)
$$

This allows us to define the mapping error in $\Delta E_{a b}^{*}$ units of one MBCCC color patch $i$ under a certain transform $\Phi_{R G B \rightarrow s R G B, m}$ as (see also eq. (3)):

$$
\begin{aligned}
& \Delta E_{a b}^{*}\left(\Phi_{R G B \rightarrow s R G B, m}, i\right)= \\
& \sqrt{\left(L_{i}^{*}-L_{S P M, i}^{*}\right)^{2}+\left(a_{i}^{*}-a_{S P M, i}^{*}\right)^{2}+\left(b_{i}^{*}-b_{S P M, i}^{*}\right)^{2}}
\end{aligned}
$$

This still doesn't provide us with a total mapping error of $\Phi_{R G B \rightarrow s R G B, m}$, but two feasible candidates are the average $\Delta E_{a b}^{*}$ error per MBCCC patch:

$$
\left\langle\Delta E_{a b}^{*}\left(\Phi_{R G B \rightarrow s R G B, m}\right)\right\rangle=\frac{1}{24} \sum_{i=1}^{24} \Delta E_{a b}^{*}\left(\Phi_{R G B \rightarrow s R G B, m}, i\right),
$$

and the maximal $\Delta E_{a b}^{*}$ error over all the 24 MBCCC patches, $\max \left(\Delta E_{a b}^{*}\left(\Phi_{R G B \rightarrow s R G B, m}\right)\right)$. The transforms resulting from the non-linear minimization of both these criteria will be notated as $\Phi_{R G B \rightarrow s R G B, m}^{a v g}$ and $\Phi_{R G B \rightarrow s R G B, m}^{\max }$ respectively.

The non-linear minimization itself is performed by the Nelder-Mead simplex algorithm [19], using the linear leastsquares solution $\Phi_{R G B \rightarrow s R G B, m}^{L L S Q}$ as an initial guess. The simplex algorithm is an iterative method and therefore much slower than the singular-value decomposition used to solve eq. (12). This is especially true for the higherorder transforms and when minimizing the highly nonlinear $\max \left(\Delta E_{a b}^{*}\left(\Phi_{R G B \rightarrow s R G B, m}\right)\right)$ function. The evaluation of the transforms and the final choice of the transform for the imaging system will be reviewed when discussing experimental results.

\section{USING THE IMAGING SYSTEM}

In order to acquire an image two conditions must be fulfilled. Firstly, the imaging system must have been calibrated at some time in the past and the resulting settings, look-up table and transform, called a profile, must be stored on the system. Secondly, this profile must be checked, accepted and adjusted. If a profile is not accepted then the user is asked to recalibrate the imaging system.

\section{A. The calibration procedure}

In the calibration procedure the optimal settings, lookup table and transform of the imaging system are determined during several consecutive steps. By sequential order these are: the determination of the camera offset, the frame grabber offset, the frame grabber gain, the camera aperture, the color gains of the camera, the linearizing look-up table, and finally the transform from the unknown device-dependent imaging system $R G B$ space to the standard device-dependent $s R G B$ space. The aim of all but the last two steps is to maximize the dynamic range and resolution of the imaging system.

The calibration procedure requires some user interaction consisting mainly of the presentation of the MBCCC color 
patches to the camera, and the manual setting of the diaphragm (although this could be done automatically with a motorized lens). It shouldn't take more than 5 minutes, and, as a profile remains valid for weeks maybe months of normal operation, it doesn't need to be repeated often.

\section{A.1 The camera offset voltage}

The aim is to make sure that a totally non-reflecting object, i.e. a object with reflective luminance $Y=0$, results in $V_{G}^{c a m}=0$. Such an object can be simulated by turning the light source off, closing the lens diaphragm and putting the lens cap on. As no other parameters of the acquisition system have been properly set so far we cannot determine an aim pixel value $G^{*}$ or $G$ for the object. We therefore adopt the following scheme:

1. Make sure the subject produces a non-zero spatially averaged green pixel value $\langle G\rangle$ by setting a high frame grabber gain and offset.

2. Measure $\langle G\rangle$ in function of the setting of camera offset voltage $V_{\text {offset }}^{\text {cam }}$.

It can be seen using (6) and (9) that this will result in a curve consisting of two straight lines: one horizontal line as long as $V_{\text {off } f \text { set }}^{\text {cam }} \leq-g_{G}^{c a m} V_{\text {offset }}^{C C D}$, and one sloping line once clipping no longer occurs and $V_{\text {offset }}^{\text {cam }} \geq-V_{\text {offset }}^{C C D}$ (see fig. 4). At the setting at which the two lines intersect the offset voltage exactly compensates for the voltage resulting from the dark current, and $V_{G}^{c a m}=0$. To determine this point reliably and independently of the frame grabber settings we fit a horizontal and a sloping straight intersecting at each of the offset voltage settings through the data, and compute the fitting errors. The desired setting is the one with the lowest error (see fig. 4). We will refer to this operation as the two-line intersection method.

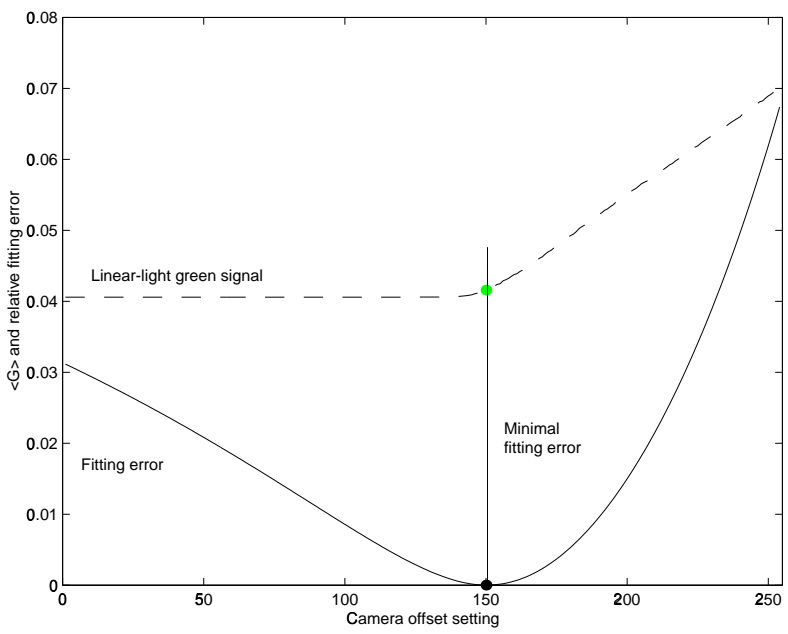

Fig. 4. The image averaged linear pixel value for the green channel in function of the camera voltage offset setting (solid line) and the corresponding fitting errors (dashed line). The setting with the smallest fitting error is indicated by the solid vertical line.

\section{A.2 The frame grabber offset voltage}

Next we determine the setting of the frame grabber offset voltage so that $\left\langle G^{*}\right\rangle=\langle G\rangle=0$. Because $V_{G}^{\text {cam }}=0$ for the simulated $Y=0$ patch, $\left\langle G^{*}\right\rangle$ is independent of the frame grabber gain $g^{f g}$. This means theoretically we only have to find the highest offset voltage setting at which $\left\langle G^{*}\right\rangle$ is zero. However, we still favor the two-line intersection method as being more robust and general. It is important to note that because the camera signal $V_{G}^{\text {cam }}$ doesn't change but is digitized with another offset voltage only $G^{*}$ has a piecewise linear relationship with the offset voltage, and not $G$ !

\section{A.3 The frame grabber gain and camera lens aperture}

In order to maximize the dynamic range of the camera we wish that $\left\langle G^{*}\right\rangle=255$ for a perfectly reflecting object ( $Y=$ $100)$, given the light source. This can be achieved in two steps. First $g^{f g}$ is set so that the maximal camera signal $V_{\max }^{c a m}$ is digitized as 255. For this the camera CCD's are saturated by fully opening the lens aperture, and measuring the highly reflecting MBCCC 'white'patch. Then $\langle G\rangle$ is measured in function of $g^{f g}$ and the two-line intersection method is again used to determine the optimal gain setting. Secondly, the continuous aperture of the lens is adjusted manually until $\left\langle G^{*}\right\rangle=255 \Gamma\left(Y_{\text {white }} / 100\right)$ for the same white patch.

\section{A.4 The camera color balance and linearizing look-up table}

The aim of these two operations is to obtain a linear response from the imaging system for series of color patches with the same chromaticity coordinates $\frac{X}{X+Y+Z}$ and $\frac{Y}{X+Y+Z}$, but different luminance $Y: R \sim Y, G \sim Y$ and $B \sim Y$. When using color patches with an almost constant reflectivity in function of wavelength (e.g. 'neutral' colors of the MBCCC), and with the added desire of optimizing the dynamic range of each color channel separately for the given light source, this becomes $R=G=B=$ $Y / 100$ (gray balancing). This process is actually a kind of normalization of RGB values with regard to the light source.

Practically the camera color dependent gain factors $g_{R}^{\text {cam }}$ and $g_{B}^{c a m}$ are adjusted first so that gray balance is obtained for the MBCCC 'white' patch using a simple bisection root finding method. Hereafter the lookup-table (LUT) is constructed in order to achieve gray balance for the 5 other MBCCC 'neutral' color patches with luminance $Y$ ranging from 3.1 to 59.1 .

\section{B. The acquisition procedure}

During the acquisition procedure the user makes sure the stored profile is still valid by comparing the current color of the MBCCC 'white' test patch with its color during the calibration procedure. If this color difference falls within certain limits, the profile is accepted and may be adjusted in order to compensate for the drift in response of the imaging system. Such an adjusted profile remains valid for 10-15 minutes, during which images can be acquired freely without any extra profile checks. 


\section{B.1 Checking and adjusting a calibration profile}

As mentioned before, once a profile is determined and stored it can be used as long as the imaging system doesn't degrade too much (aging of the light source bulb, changes in CCD sensor spectral response, etc. ...). The imaging system does, however, exhibit a short term drift in response which more or less stabilizes after about 30-40 minutes of warmup-time, see fig. 5. In order to compensate for this we

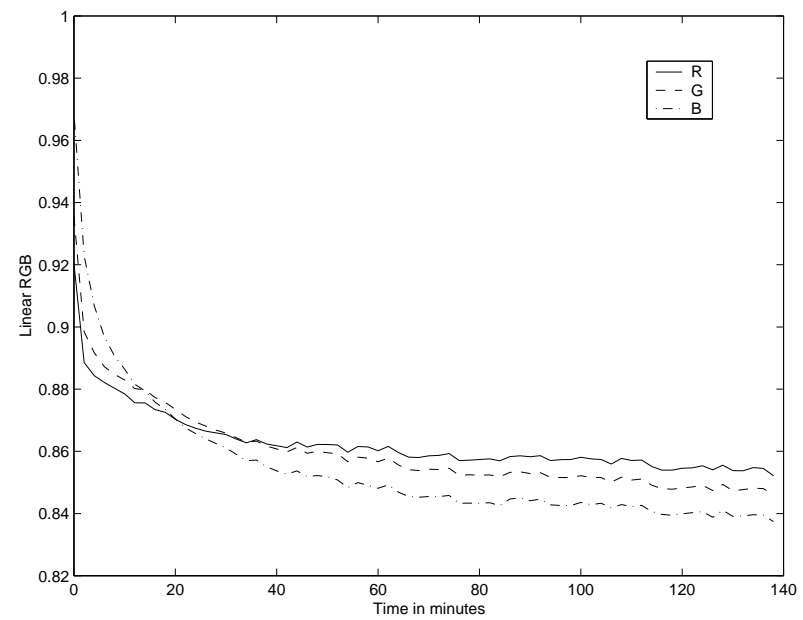

Fig. 5. The linear RGB response of the white MBCCC patch in function of the warmup-time of the imaging system and with no profile adjustment.

use the theoretical $R G B$ response of the white test patch $R=G=B=Y_{\text {white }} / 100$, see also V-A.4, to adjust some of the profile settings prior to imaging.

Normally, when changing one or more of the profile settings the linearizing LUT and transform $\Phi_{R G B \rightarrow s R G B, m}$ should be recomputed, but here we make the implicit assumption that these remain valid if the adjustments to the settings are small. This is why no attempt is made to adjust a profile if the white test patch measured during the profile check has a color difference $\Delta E_{a b}^{*} \leq 2$ with its value at calibration. That the assumption for the LUT and the transform are reasonable can be seen in fig. 6 , where $\Delta E_{a b}^{*}$ of the white patch with its value at calibration, before and after profile adjustment, is plotted. A complete proof would consist in measuring the average $\Delta E_{a b}^{*}$ of the whole set of MBCCC patches before and after adjustment...

The image used for checking the profile is also used for multiplicative shading correction. This corrects for spatial inhomogeneities in the lighting and in the efficiency of the CCD array.

\section{B.2 Acquiring an image}

After the calibration profile is checked, accepted and adjusted images may be acquired freely for about 10-15 minutes without rechecking the profile. These images are transformed to the gamma-corrected $s R^{*} G^{*} B^{*}$ space and stored, see fig. 3. This is called output rendering and should provide a fairly realistic image on any modern CRT-based monitor which has its white-point set at $6500 \mathrm{~K}$ [20]. Fig.

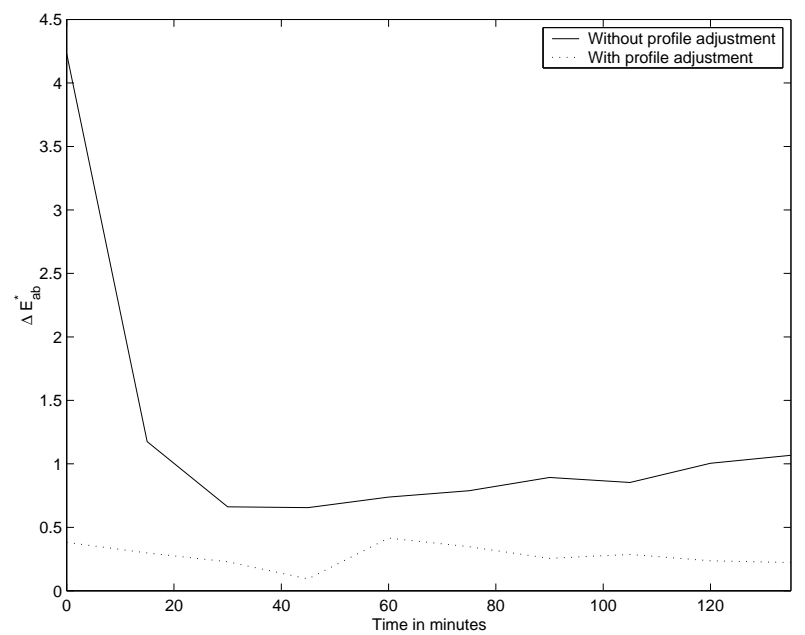

Fig. 6. The $\Delta E_{a b}^{*}$ color differences of the white MBCCC patch with its $L^{*} a^{*} b^{*}$ value at calibration in function of the warmup-time of the imaging system and with and without profile adjustment.

7 shows a clinical and a dermatoscopic image of a kind of mole called a junction naevus, acquired with the imaging system.

\section{EXPERIMENTAL RESULTS AND DISCUSSION}

\section{A. Precision}

With precision we mean the reproducibility of measurements, or the way repeated measurements are spread around the average of those measurements. Precision will be important for any quantitative measurement of image color characteristics. We can distinguish several types of precision: short-term precision when making several consecutive measurements of the same color, medium-term or profile precision when comparing measurements made under one calibration profile, and long-term or inter-profile precision when talking about the agreement between measurements made under different calibration profiles.

Short term precision based on 20 consecutive measurements of the MBCCC 'white' patch was very good: $\left\langle\Delta E_{a b}^{*}\right\rangle=0.04$, with $\Delta E_{a b}^{*}<0.1$. The results for the medium-term and long-term precision can be seen in fig. 8 . Here the average error and maximal error in $\left\langle\Delta E_{a b}^{*}\right\rangle$ units was computed for each MBCCC patch with regard to the average sample $L^{*} a^{*} b^{*}$ value of that patch (10 and 9 measurements respectively). To simulate possible long-term changes in the imaging system, the color temperature of the light source was modified for half of the profiles. There was no noticeable difference in precision between profiles for the normal and modified imaging system. The average and maximal errors over all the MBCCC patches for medium-term and long-term precision are $\left\langle\Delta E_{a b}^{*}\right\rangle=0.34$ with $\Delta E_{a b}^{*}<1.2$, and $\left\langle\Delta E_{a b}^{*}\right\rangle=0.30$ with $\Delta E_{a b}^{*}<1.2$ respectively. The fact that the long-term precision is slightly better than the medium-term precision might be explained by the fact that any measurements for the long-term precision were made directly after the profile was determined, thereby avoiding the drift problems outlined in V-B.1. 


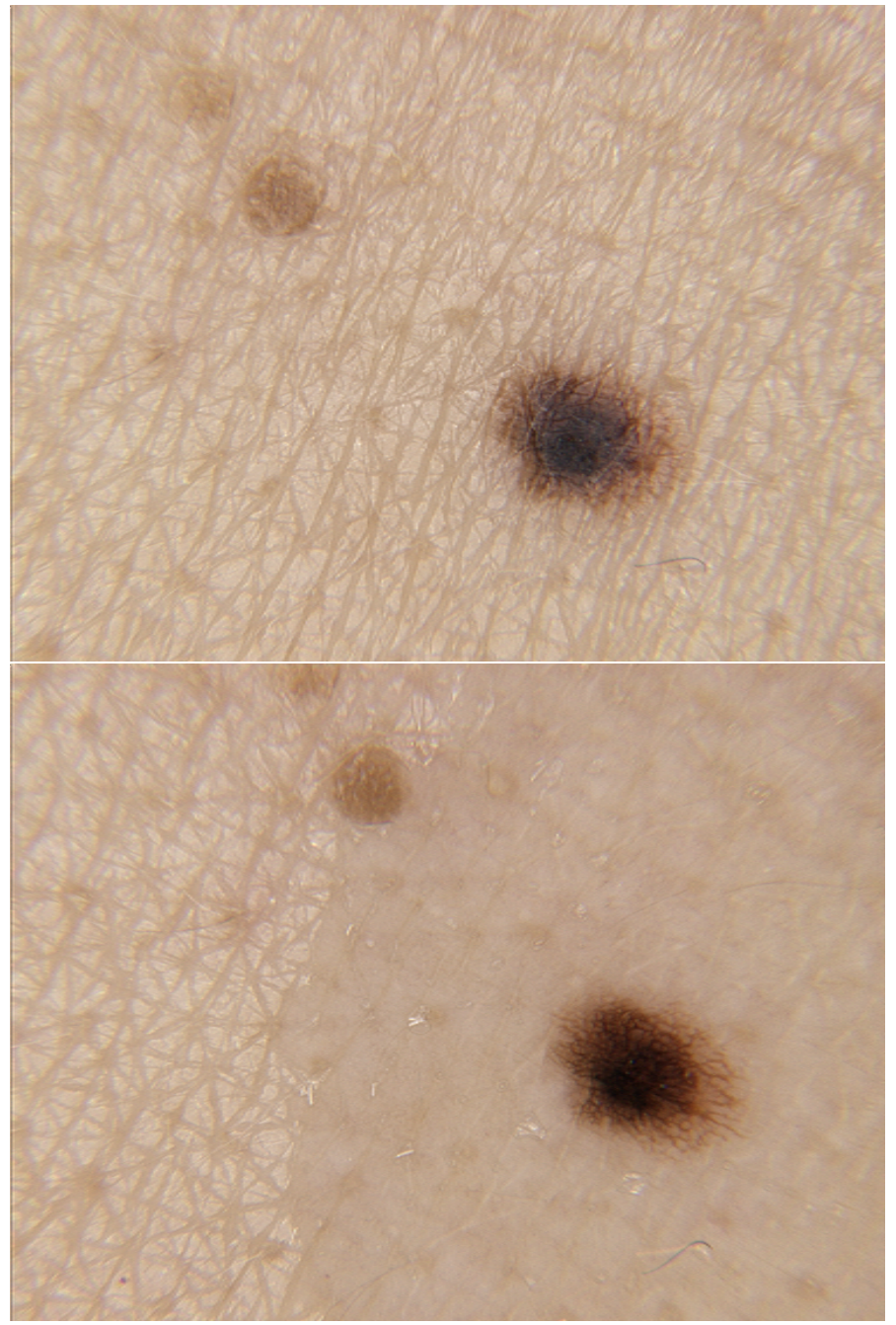

Fig. 7. A junction naevus shot with the imaging system, both clinically (top) and dermatoscopically (bottom). Notice, how on the bottom image the oil used for dermatoscopy did not spread properly.

\section{B. Accuracy}

With accuracy we mean the way in which measurements of colors made with the imaging system are close to the measurements made by a reference instrument, a spectrophotometer in this case. To quantify the accuracy we compute the average and maximal $\Delta E_{a b}^{*}$, although now over a set of test patches different from the MBCCC patches used in computing the transform $\left.\Phi_{R G B \rightarrow s R G B, m}, i\right)$. This test set consists of 15 plastic and paper patches, as well as 12 skin areas (normal Caucasian and Asian skin, moles, pimples, ...) from human volunteers. The samples are all measured with the SPM as well as imaged with the imaging system. Pixels in these images are averaged over an area roughly corresponding to the measurement area of the SPM (circle of $5 \mathrm{~mm}$ diameter). This is necessary because the skin areas are not always completely uniform in color. Because the precision is expected to be much higher than the accuracy there is no need to average image measurements over several acquisi-
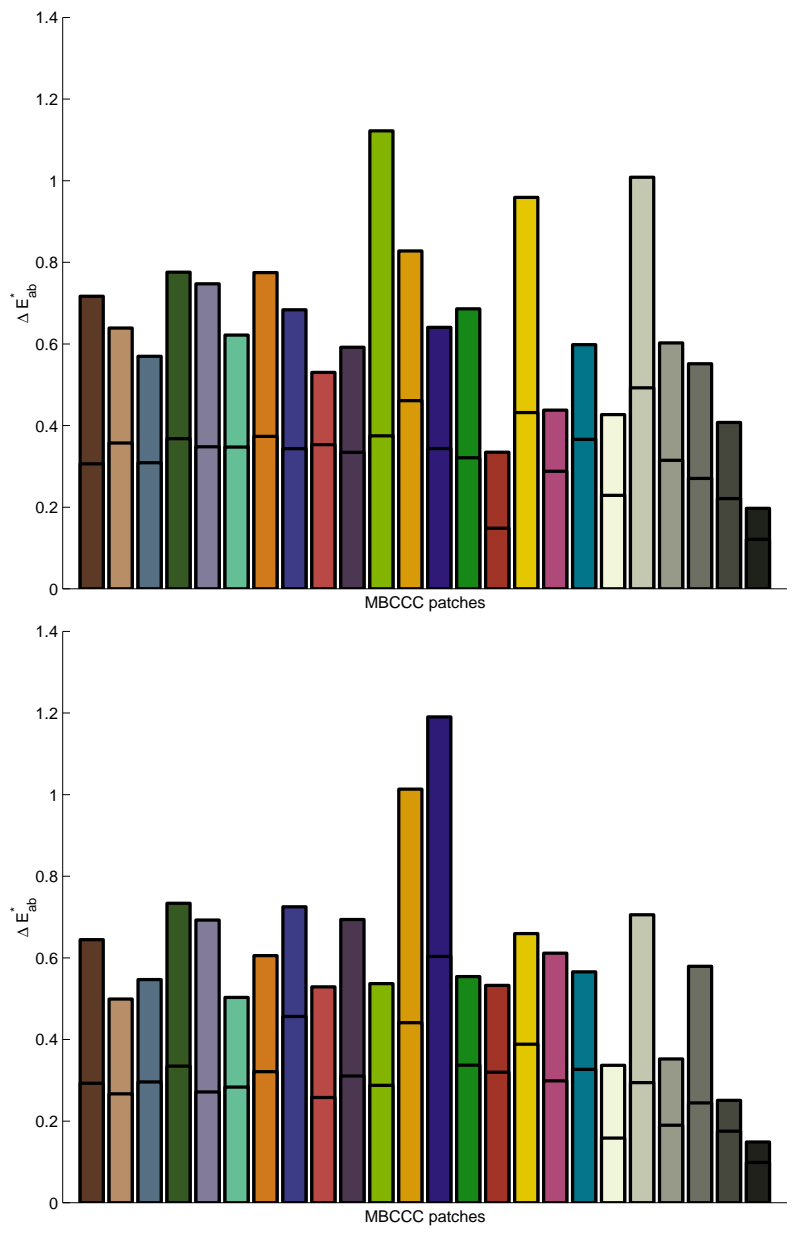

Fig. 8. Average and maximal $\Delta E_{a b}^{*}$ error of the 10 measurements of the MBCCC patches with regards to their average determined in CIE $L^{*} a^{*} b^{*}$ space. Top graph gives results under one calibration profile, i.e. medium-term precision, and bottom graph under several calibration profiles, i.e. long-term precision. Bar colors are similar to the those of the MBCCC patches they represent.

tions. It is clear the accuracy of the whole imaging system depends mainly on the performance of the different transforms $\Phi_{R G B \rightarrow s R G B, m}, m=3,6,8,9,11$ (see fig. 9). The performance of the different transforms on the MBCCC patches used in computing them is generally as expected: diminishing errors for an increasing number of terms, and superiority of the non-linearly optimized transforms. Notice how trying to minimize the maximal patch error results in a strongly increased average error per patch.

Surprisingly the linear LLSQ solution $\Phi_{R G B \rightarrow s R G B, 3}^{L L S Q}$ has the lowest average $\Delta E_{a b}^{*}$ at 6.21 and the second lowest maximal $\Delta E_{a b}^{*}$ at 13.31 over the test set. Very probably this is due to the limited number of patches used in computing the transforms, leading to a very sparse sampling of the $R G B$ and $s R G B$ color spaces. This hardly affects the linear transforms which have very good generalizing properties, but may lead to uncontrolled, oscillating behavior between the sample points for higher-order transforms. It is unclear why the $\Gamma_{3}$ transform optimized for average $\Delta E_{a b}^{*}$ does not perform better than its SVD counterpart.

Fig. 10 shows the $\Delta E_{a b}^{*}$ errors of the individual patches of 

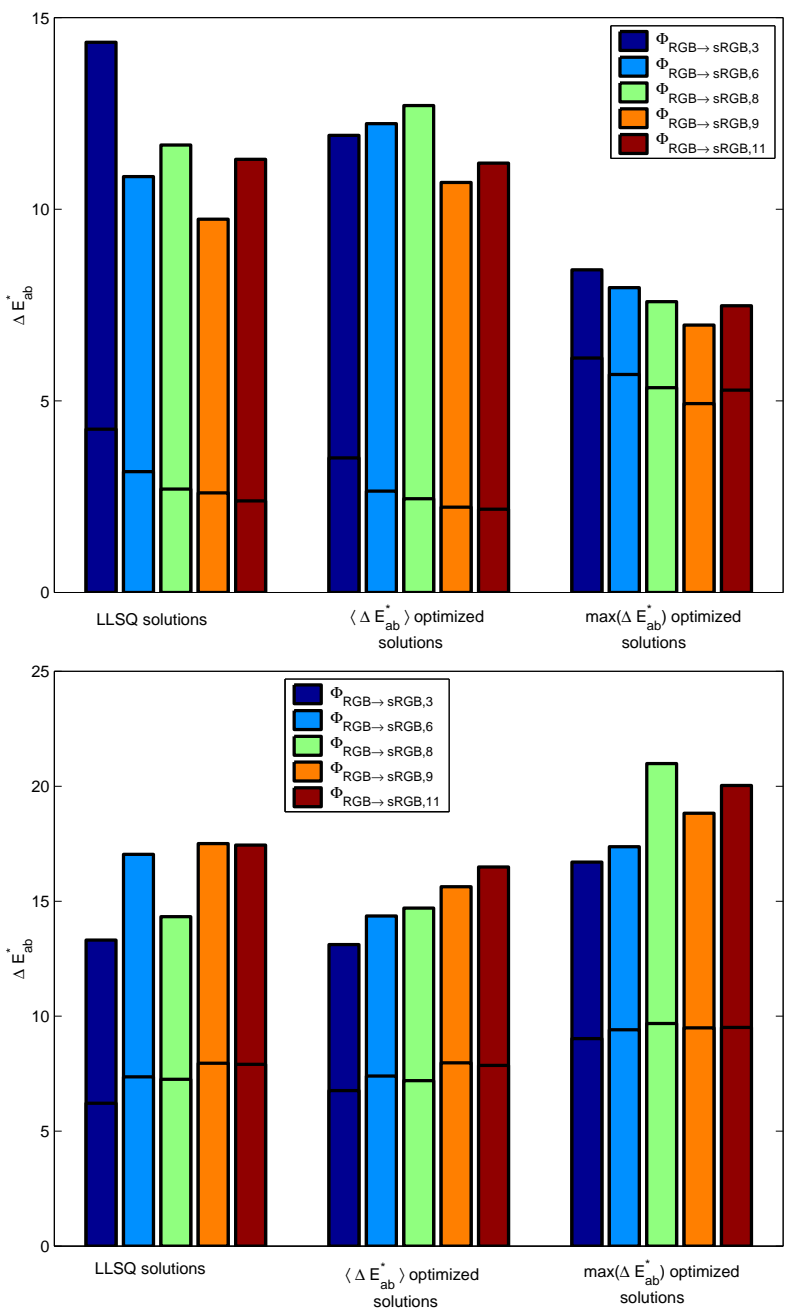

Fig. 9. Average and maximal $\Delta E_{a b}^{*}$ error of the imaging system measurements of the MBCCC patches (top) and the test set (bottom) with their SPM measurements, for all the LLSQ, average and maximal $\Delta E_{a b}^{*}$ optimized transforms $\Phi_{R G B \rightarrow s R G B, m}, m=$ $3,6,8,9,11$.

the test set for $\Phi_{R G B \rightarrow s R G B, 3}^{L L S Q}$. It is important to note here that the 'skin' targets (bars on the right) show the same range of errors as the paper and plastic targets (bars on the right), even if their spectra maybe completely different from the ones used to determine the transform. This shows that the machine-human metamerism problem is probably not an issue for skin imaging.

\section{CONCLUSIONS AND FUTURE WORK}

We have proposed a color imaging system which allows colorimetrically consistent acquisition of images. This is possible by calibrating the system and storing the resulting settings in a profile. Several types of device-dependent to device-independent color space polynomial transforms were tested, including some optimized in perceptually uniform color spaces. Determining a profile takes a little user input and shouldn't take more than 5 minutes. Such a profile remains valid for weeks or even months of normal operation of the imaging system, so it only needs to be performed once in a while. Before acquiring images a profile

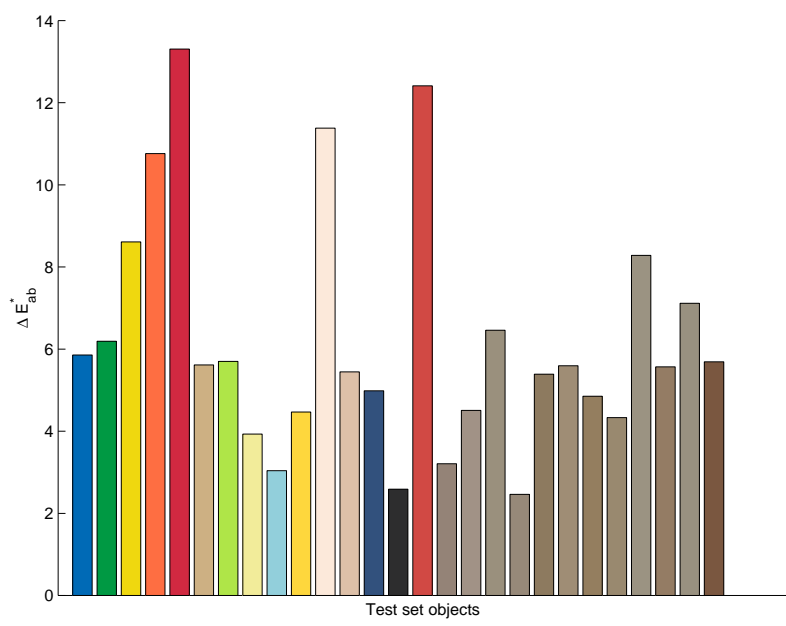

Fig. 10. The $\Delta E_{a b}^{*}$ errors of the test set of 15 plastic and paper patches (bars on the left) and 12 in vivo skin areas (bars on the right) between the SPM and the imaging system measurements using the LLSQ linear transform $\Phi_{R G B \rightarrow s R G B, 3}^{L L S Q}$. Bar colors are similar to the those of the test patches they represent

is checked and adjusted using just one white color patch. Images may then be acquired for a period of about 10 to 15 minutes before a new profile check is necessary. The imaging system has good precision and fair accuracy. Images are stored in a standard color space with known primaries and white point, and as such can be exchanged and compared with other images defined in the same color space, even if acquired by another imaging system. They result in realistic viewing on CRT monitors without the need to convert them to another color space. The use of human vision related color spaces with their ability to compute perceptual color differences provides a proper framework for the development of segmentation and classification methods, as well as ensuring their wider applicability and possible outside validation.

\section{ACKNOWLEDGMENT}

This project is funded by the FWO in Belgium, ref. F7801

\section{REFERENCES}

[1] N. Cascinelli et al., "A possible tool for clinical diagnosis of melanoma: The computer," Journal of the American Academy of Dermatology, vol. 16, no. 2, Part 1, pp. 361-367, 1987.

[2] J. L. Stone, R. L. Peterson, and J. E. Wolf, "Digital imaging techniques in dermatology," Journal of the American Academy of Dermatology, no. 5, part 1, pp. 913-917, 1990.

[3] R. Kenet et al., "Clinical diagnosis of pigmented lesions using digital epiluminescence microscopy," Arch. of Dermatology, vol. 129 , pp. $157-174,1993$.

[4] W. Stolz et al., "Improvement of monitoring of melanocytic skin lesions with the use of a computerized acquisition and surveilland with a skin surface microscopic television camera," Journal of the American Academy of Dermatology, vol. 35, no. 2, Part 1, pp. 202-207, 1996.

[5] T. Schindewolf et al., "Evaluation of different image acquisition techniques for a computer vision system in the diagnosis of malignant melanoma," Journal of the American Academy of Dermatology, vol. 31, no. 1, pp. 33-41, 1994.

[6] M. Nischik et al., "Analysis of skin erythema using true-color images," IEEE Trans. On Medical Images, vol. 16, no. 6, pp. 711$715,1997$. 
[7] G. E. Healey and R. Kondepudy, "Radiometric CCD camera calibration and noise estimation," IEEE Transcations on Pattern Analysis and Machine Intelligence, vol. 16, no. 3, pp. 267-276, 1994.

[8] Y.-C. Chang and J. F. Reid, "RGB calibration for color image analysis in machine vision," IEEE Transactions on Image Processing, vol. 5, no. 10, pp. 1414-1422, 1996.

[9] B. A. Wandell, "The synthesis and analysis of color images," IEEE Transactions on Pattern Analysis and Machine Intelligence, vol. PAMI-9, no. 1, pp. 2-13, 1987.

[10] D. Slater and G. Healey, "The illumination-invariant recognition of $3 \mathrm{~d}$ objects using local color invariants," IEEE Transactions on Pattern Analysis and Machine Intelligence, vol. 18, no. 2, pp. 206-210, 1996.

[11] G. Finlayson, "Color in perspective," IEEE Transactions on Pattern Analysis and Machine Intelligence, vol. 18, no. 10, pp. 1034-1038, 1996.

[12] G. D. Finlayson and M. S. Drew, "White-point preserving color correction," in Fifth Color Imaging Conference: Color Science, Systems and Applications, pp. 258-261, IS\&T, SID, 1997.

[13] E. Boldrin, P. Campadelli, and R. Schettini, "Learning color appearance models," in Fifth Color Imaging Conference: Color Science, Systems and Applications, pp. 173-176, IS\&T, SID, 1997.

[14] P. M. Hubel, J. Holm, G. D. Finlayson, and M. S. Drew, "Matrix calculations for digital photography," in Fifth Color Imaging Conference: Color Science, Systems and Applications, pp. 105111, IS\&T, SID, 1997.

[15] H. Kang, Color Technology for Electronic Imaging Devices. SPIE Optical Engineering Press, 1997.

[16] G. Wyszecki and W. Stiles, Color Science: Concept and Methods, Quantitative Data and Formulae. John Wiley \& Sons, second edition ed., 1982.

[17] R. Hunt, The Reproduction of Colour. Fountain Press, fifth edition ed., 1995.

[18] G. Holst, $C C D$ arrays, Cameras and Displays. SPIE Optical Engineering Press, 1996.

[19] W. H. Press, Numerical Recipes in C: The Art of Scientific Computing. Cambridge Univ. Press, 1993.

[20] J. Holm, "Issues relating to the transformation of sensor data into standard color spaces," in Fifth Color Imaging Conference: Color Science, Systems and Applications, pp. 290-295, IS\&T, SID, 1997. 